\title{
Open Access Journal of Urology \& Nephrology
}

\section{Acute Kidney Injury-Recent Thoughts in Diagnosis and Management}

\section{Rashid HU}

Department of Nephrology, Kidney Foundation hospital and research Institute,

Mirpur, Dhaka, Bangladesh

\section{Review Article}

Volume 2 Issue 1

Received Date: September 24, 2016

Published Date: January 04, 2017

*Corresponding author: Harun Ur Rashid, Professor and Chief Consultant, Kidney Foundation Hospital and Research Institute, Road 1, Section-2, Mirpur, Dhaka-1616, Bangladesh, Tel: 88029024865/ 8801819247299; E-mail: rashid@bolonline.com

\section{Introduction}

Acute Kidney Injury (AKI) has become a global health challenge with an estimated incidence of 13.3 million every year. In developing countries, the annual incidence is 11.3 million cases.

It causes 1.7 million death per year globally; of them, 1.4 million in low and middle-income countries. About 15$20 \%$ adults admitted to hospital in developed countries develop AKI whereas in developing countries the incidence ranges from $25-30 \%$.

\section{Keywords: Acute Kidney Injury; acute renal failure}

\section{History of AKI}

William Heberden first described acute renal failure as "Ischemic renalis" in 1802. One hundred years later, William Osler in text book of medicine described "acute renal failure" as "acute Bright's disease" [1,2].

Homer W, Smith was first described the term "Acute renal failure" in his text book "The Kidney - structure and function in health and disease in 1951 [3].

Since 1951, there was no consensus on the diagnostic criteria or clinical definition of ARF. Kellum et al 2002 review the literature and found at least 35 definitions in the literature.

The acute dialysis quality initiative (ADQI) group in 2004, $1^{\text {st }}$ developed a system for diagnosis and classification of a broad range of acute impairment of kidney function through a broad consensus of experts in 2004 [4].

The characteristic of this system are risk, injury and failure, and the two outcome classes, loss and end stage renal disease. The three severity grades are defined on the basis of the changes in Serum creatinine and Urine output. The two-outcome criteria, loss and ESKD are defined by the duration of loss of kidney function. The criteria for diagnosis of AKI by RIFLE classification.

More recently, the acute kidney injury network (AKIN), an international network of AKI researchers, organized a summit of nephrology and critical care societies from around the world, endorsed the RIFLE criteria. With a small changes in Serum Creatinine $(0.3 \mathrm{mg} / \mathrm{d}$ or $\geq 26.5$ $\mu \mathrm{mol} / \mathrm{l}$ ) when they occur within 48 hours period. Two recent studies in USA and Europe validated these modified criteria. Thakar et al. found that increased severity of AKI was associated with an increased risk of death independent of co morbidity $[4,5]$.

AKI should be differentiated for acute kidney disease, where there are changes of structure and functions and chronic kidney disease.

\section{Classification of AKI}

AKI is classified as extrinsic or Pre-renal, Intrinsic or renal and Post renal or obstructive causes. The primary causes of Pre-renal are hypo-volemic state i.e. gastroenteritis, hemorrhage. Edematous state i.e. congestive heart failure, cirrhosis of liver and nephrotic 


\section{Open Access Journal of Urology \& Nephrology}

syndrome. Hypotension state i.e. sepsis, cardiogenic shock and arrhythmias and renal hypo-perfusion i.e. use of nonsteroid anti-inflammatory drugs, use of anti-hypertensive and anti-proteinemic like ACE-inhibitor, angiotensin receptor blocker and hepato-renal cause.

In developing countries, water borne disease accounts for $>2.2$ million death and 82 million disability adjusted life years. Water borne infection can cause kidney injury by causing diarrhoea, sulmonellosis, shigellosis and cholera. Vector borne disease like Falciparum malaria and Dengue fever are important causes of AKI and so leptospirosis and Schistosomiasis.

The intrinsic causes of AKI are glomerular disease, tubular injury; acute interstitial nephritis caused by drugs and infections and vascular disease.

The post renal causes are renal stone disease, urethral stricture, prostatic hypertrophy or malignancy, bladder tumor, pelvic malignancy and radiation fibrosis.

\section{How to assess the risk of AKI}

The factors that increase the susceptibility of AKI are dehydration or volume depletion, advance age, presence of underlying CKD, presence of chronic disease, like diabetes mellitus, heart, lung, liver disease and cancer.

\section{How to diagnose AKI}

The diagnosis of AKI needs careful history of patients and thorough clinical examination and laboratory investigations. Urine is collected for routine and microscopic examination for red blood cell, white blood cell and casts. Proteinuria, in presence of RBC and cast usually include glomerular disease.

Estimation of blood for urea, creatinine and electrolytes are essential for all cases. If glomerular disease is suspected, complement C3, C4, anti-nuclear factor, anti-ds DNA and anti-nutrophilic cytoplasmic antibody are valuable. Ultra-sound of kidney and plain Xray KUB is also necessary to exclude obstructive nature of disease.

New biomarkers are helpful in post surgical causes of AKI particularly heart surgery.

The biomarkers are Cystatine-C, kidney injury molecule- 1(KIM-1), Neutrophil gilatenase associated lipocalin (NGAL), Interleukin 18 and Urine-N-acetyl $\beta$-Dglucosaminidase.
Coca et al reviewed the literature for early biomarkers of AKI from 2000-2007 and considered that serum CystatinC, Urinary NGAL, urinary IL-18 and KIM-1 is helpful for early diagnosis of AKI while Urinary $\mathrm{N}$-acetyl $\beta$-Dglucosaminidase, KIM-1 can predict severity risk for AKI [6].

\section{Evaluation and general management of patients with AKI}

The patients should be evaluated promptly to determine the cause. Each patient should be monitor regularly with serum creatinine, electrolyte and urine output. All patients should be evaluated for 3 months for resolution or worsening of pre-existing CKD.

\section{Management of hypotension and shock}

Careful titration of fluid is required for patients with hypotension and shock. If the cause is diarrhoea, oral rehydration salt is very useful for children and infant. In young and older age groups intravenous saline, 0.9\% along with potassium and bi carbonate are needed [7-9].

In ICU patients, isotonic saline may cause fluid overload and in that case, $4 \%$ albumin can be considered.

After correction of dehydration, if blood pressure is low dopamine or vasopressin can be used to maintain blood pressure. It is useful in septic shock, burns and liver failure but can be hazardous in cardiogenic shock.

Blood sugar level is altered in patients with AKI and tight glycaemic control and nutritional support are needed. The blood sugar is maintained between 80-110 $\mathrm{mg} / \mathrm{dl}$ and total calorie intake is $20-30 \mathrm{KCal} / \mathrm{kg} /$ day.

Protein is used liberally at $0.8-1.0 \mathrm{gm} / \mathrm{kg} /$ day in noncatabolic patients, where as in hyper-katabolic AKI protein is increased to $1.0-1.5 \mathrm{~g} / \mathrm{kg} /$ day [10].

\section{Role of diuretics in AKI}

Diuretics are used in most of the patients with AKI, if oliguria or anuria is persisted for several hours.

However, Ho and Power reviewed the literature and did not find any beneficial effect of diuretic in patients with early recovery of AKI. Diuretic only convert oliguria to non-oliguria and promote earlier dialysis but there is no effect on survival benefit. However diuretics can be used in patients who are fluid over loaded [11,12]. 


\section{Open Access Journal of Urology \& Nephrology}

\section{Role of vasodilator therapy}

Low dose dopamine has been using longtime for early recovery of AKI. Kellum and Decor found no beneficial effect in early recovery of AKI. While Friedrich et al, found that instead of beneficial effect if may cause harm from low dose dopamine. It may cause tachy-arrhythmias and myocardial ischaemia, decrease intestinal blood flow, and can suppress T-cell function.

The majority of trails with low dose dopamine have been small with end of questionable clinical significant. Recent data suggest that the renal vasodilator effect of dopamine found in healthy populations is not preserved in patients with AKI. Using Doppler ultrasound found that dopamine significantly increased renal vascular resistance in AKI patients and found no benefit of dopamine for prevention or therapy of AKI in an adequetly powered meta-analysis and Marik found no benefit in a systematic review $[13,14]$

\section{When to start renal replacement therapy in AKI}

The optimum timing of dialysis in AKI is based on clinical feature of volume overloud and biochemical features of salute imbalance such as azotemia, hyperkalemia and severe acidosis. Delay of dialysis is made when recovery is suspected on their own however there are risk of dialysis in AKI such as hypotension, arrhythmia, membrane bio in compability and complication of vascular access and anticoagulant administration [15-17].

Controversy exists as to which is the optimal RRT modality for patients with AKI. The choice of initial modality of RRT is based on availability of and experience with a specific treatment and on the patients hemodynamic status.

However intermittent haemodialysis and CRRT is mostly dependant on experience and haemodynamic status of patients, coagulation problems, although end result does not significantly vary between the two modes of therapy $[18,19]$

Experience with PD in AKI is limited except pediatric setting and in region with limited resource. Slow low efficiency dialysis (SLED) is as efficient as CRRT and valuable in low resource setting.

\section{Prognosis of AKI}

The prognosis of AKI dependent on underline cause, duration of disease, presence of multiple organ failure, hypotension, stage of AKI, older age and need for dialysis.

\section{Prevention of AKI}

The prevention of AKI is dependent on general physician, nephrologist, along with government and public health support.

Clinician need early recognition, prompt diagnosis and initiation of early treatment while government has immense role to improve water supply, improvement of sanitation, control of flood, program for infection control and improvement of maternal and child health.

Avoidance of inadvertent use of drugs particularly antibiotic and analgesic is also very important in hospital and out patients.

Appropriate knowledge is needed for appropriate dose of drugs and duration of treatment.

\section{References}

1. Eknoyan G (2002) Emergence of the concept of acute renal failure. Am J Nephrol 22(2-3): 225-230.

2. Davies F, Weldon R (1917) A contribution to the study of "war nephritis". Lancet 190: 118-120.

3. Kellum JA, Levin N, Bouman C, Lameire N (2002) Developing a consensus classification system for acute renal failure. Curr Opin Crit Care 8(6): 509-514.

4. Bellomo R, Ronco C, Kellum JA, Mehta RL, Palevsky P (2004) Acute renal failure -definition, outcome measures, animal models, fluid therapy and information technology needs: the Second International Consensus Conference of the Acute Dialysis Quality Initiative (ADQI) Group. Crit Care 2004; 8(4): R204-212.

5. Thakar CV, Christianson A, Freyberg R, Almenoff P, Render ML (2009) Incidence and outcomes of acute kidney injury in intensive care units: a Veterans Administration study. Crit Care Med 37(9): 25522558. 


\section{Open Access Journal of Urology \& Nephrology}

6. Coca SG, Yalavarthy R, Concato J, Parikh CR (2008) Biomarkers for the diagnosis and risk stratification of acute kidney injury: A systematic review. Kidney Int; 73(9) 1008-1016.

7. Bellomo R, Wan I, May CN (2009) Vasoactive drugs and acute renal failure. In: Ronco C, Bellomo R, Kellum I (Eds) Critical Care Nephrology 2nd (Edn). Saunders Elsevier, Philadelphia, PA, Pp: 416-419.

8. Finfer S, Jones DA (2009) Crystalloids and colloids. In: Ronco C, Beliomo R, Kellum J (Eds) Critical Care Nephrology $2^{\text {nd }}(E d n)$ Saunders Elsevier, Philadelphia, PA, pp: 571-575.

9. Finfer S, Bellomo R, Boyce N, French J, Myburgh J, et al. (2004) A comparison of albumin and saline for fluid resuscitation in the intensive care unit. $\mathrm{N}$ Engl J Med 350(22): 2247-2256.

10. Van den Berghe G, Wouters P, Weekers E, Verwaest C, Bruyninckx F, et al. (2001) Intensive insulin therapy in the critically ill patients. $\mathrm{N}$ Engl J Med 345(19): 1359-1367.

11. Ho KM, Sheridan DJ (2006) Meta-analysis of frusemide to prevent or treat acute renal failure. BMJ 26: 333: 420 .

12. Ho KM, Power BM (2010) Benefits and risks of furosemide in acute kidney injury. Anaesthesia 65(3): 283-293.
13. Friedrich JO, Adhikari N, Herridge MS, Beyene J (2005) Meta-analysis: Low-dose dopamine increase urine output but does not prevent renal dysfunction or death. Ann Intern Med 142(7): 510-524.

14. Murray PT (2006) Fenoldopam: renal -dose dopamine. Crit Care Med 34(3): 910-911.

15. Palevsky PM, Baldwin I, Davenport A, Goldstein S, Paganini E (2005) Renal replacement therapy and the kidney: minimizing the impact of renal replacement therapy on recovery of acute renal failure. Curr opin Crit Care 11(6): 548: 554.

16. Kraut JA, Kurtz I (2008) Toxic alcohol ingestions: clinical features, diagnosis, and management. Clin J Am Soc Nephrol 3(1): 208-225.

17. Conger JD (1975) A controlled evaluation of prophylactic dialysis in post-traumatic acute renal failure. J Trauma 15(12): 1056-1063.

18. Pannu N, Klarenbach S, Wiebe N, Manns B, Tonelli M (2008) Renal replacement therapy in patients with acute renal failure: a systematic review. JAMA 299(7): 793-805.

19. Srisawat N, Lawsin I, Uchino S (2010) Cost of acute renal replacement therapy in the intensive care unit: results from the Begjnning and Ending Supportive Therapy for the kidney (BEST Kidney) study. Crit Care 14: R46. 